\title{
Pten-mediated Gsk3 $\beta$ modulates the naïve pluripotency maintenance in embryonic stem cells
}

\author{
Wuming Wang ${ }^{1,2}$, Gang Lu ${ }^{1,2}$, Xianwei Su ${ }^{1,2,3}$, Chengcheng Tang ${ }^{1}$, Hongjian Li ${ }^{1,2,3}$, Zhiqiang Xiong ${ }^{2,3}$, \\ Chi-Kwan Leung ${ }^{1,2,3}$, Man-Sze Wong $\mathbb{B}^{1,2}$, Hongbin Liu ${ }^{1,2}$, Jin-Long Ma ${ }^{1,2,3}$, Hoi-Hung Cheung ${ }^{1}$, Hsiang-Fu Kung ${ }^{1,2}$, \\ Zi-Jiang Chen ${ }^{1,2,4}$ and Wai-Yee Chan $\mathbb{1}^{1,2}$
}

\begin{abstract}
Mouse embryonic stem cells (ESCs) are isolated from the inner cell mass of blastocysts, and they exist in different states of pluripotency-naïve and primed states. Pten is a well-known tumor suppressor. Here, we generated Pten ${ }^{-1-}$ mouse ESCs with the CRISPR-Cas9 system and verified that Pten ${ }^{-1}$ ESCs maintained naive pluripotency by blocking Gsk3 $\beta$ activity. Serum/LIF and $2 i$ (MAPK and GSK3 inhibitors) conditions are commonly used for ESC maintenance. We show that the Pten-inhibitor SF1670 contributed to sustaining mouse ESCs and that Pten activation by the S380A, T382A, and T383A mutations (Pten-A3) suppressed the pluripotency of ESCs. The in vivo teratoma formation ability of SF1670treated ESCs increased, while the Pten-A3 mutations suppressed teratoma formation. Furthermore, the embryoid bodies derived from Pten-deficient ESCs or SF1670-treated wild-type ESCs showed greater expression of ectoderm and pluripotency markers. These results suggest that Pten-mediated Gsk3 $\beta$ modulates the naïve pluripotency of ESCs and that Pten ablation regulates the lineage-specific differentiation.
\end{abstract}

\section{Introduction}

Embryonic stem cells (ESCs) are pluripotent stem cells derived from the inner cell mass of the early mammalian embryo $^{1-3}$. ESCs possess the ability to self-renew and to differentiate into cells representative of all three embryonic germ layers ${ }^{4}$. In both mice and humans, ESCs exist in different pluripotent states such as the naïve and primed states, and these two states of pluripotency are distinguished by unique molecular and cellular features ${ }^{5}$. Self-renewing, pluripotent, and lineage primed states have been defined by multiple methodologies, including the recently reported method of single-cell expression profiling .

Correspondence: Gang Lu (lugang@cuhk.edu.hk) or Wai-Yee Chan (chanwy@cuhk.edu.hk)

${ }^{1}$ CUHK-SDU Joint Laboratory on Reproductive Genetics, School of Biomedical Sciences, The Chinese University of Hong Kong, Hong Kong, China

${ }^{2}$ National Research Center for Assisted Reproductive Technology and

Reproductive Genetics, Jinan 250001, China

Full list of author information is available at the end of the article.

Edited by I. Amelio
Small-molecule inhibitors have been used to maintain the pluripotency of mouse and primate $\mathrm{ESCs}^{7,8}$. Mouse ESCs are maintained in a ground state of pluripotency in the presence of MAP kinase/ERK kinase (MEK) and glycogen synthase kinase 3 (GSK3) inhibitors (2i, PD0325901 and CHIR99021) that can increase the expression of pluripotency factors in $\mathrm{ESCs}^{9}$, and the cytokine leukemia inhibitory factor (LIF) drives self-renewal of mouse ESCs by activating the transcription factor $\mathrm{STAT} 3^{10,11}$. Wnt/ $\beta$-catenin signaling is important in maintaining the pluripotency of mouse ESCs by inhibiting GSK3 with CHIR99021 7 . BIO (6-bromoindirubin-3'-oxime), a GSK3-specific pharmacological inhibitor, inhibits the differentiation of ESCs and activates the Wnt pathway to maintain the expression of the pluripotency sustaining factors Rex1, Oct4, and $\mathrm{Nanog}^{7}$.

The Pten gene is a well-known tumor suppressor and is essential for embryonic development. Pten ${ }^{-1-}$ homozygous embryos exhibit lethality with defective placentation and death by embryonic day (E) $7.5^{12}$. Pten controls the renewal and differentiation of neural and glioma

\section{(c) The Author(s) 2020}

(c) Open Access This article is licensed under a Creative Commons Attribution 4.0 International License, which permits use, sharing, adaptation, distribution and reproduction cc) in any medium or format, as long as you give appropriate credit to the original author(s) and the source, provide a link to the Creative Commons license, and indicate if changes were made. The images or other third party material in this article are included in the article's Creative Commons license, unless indicated otherwise in a credit line to the material. If material is not included in the article's Creative Commons license and your intended use is not permitted by statutory regulation or exceeds the permitted use, you will need to obtain permission directly from the copyright holder. To view a copy of this license, visit http://creativecommons.org/licenses/by/4.0/. 
stem/progenitor cells ${ }^{13,14}$, and Pten $^{-/}$ESCs form aberrant embryoid bodies (EBs) and show an altered differentiation potential ${ }^{15}$. PTEN is a phosphatase that dephosphorylates phosphatidylinositol-3, 4, 5-trisphosphate $\left(\mathrm{PIP}_{3}\right)$, and it negatively regulates the phosphoinositide-3 kinase (PI3K) signaling pathway to inhibit AKT activity ${ }^{16}$. PI3K signaling has recently been shown to manipulate mammalian preimplantation embryogenesis ${ }^{17}$, and inhibition or disruption of the PI3K/AKT pathway results in loss of pluripotency and viability of ESCs and promotes the differentiation of $\mathrm{ESCs}^{18,19}$.

In this study, we established the Pten ${ }^{-1-}$ and Pten-A3 mutant (S380A, T382A, and T383A) ESC lines by using the CRISPR-Cas9 system and aimed to determine the roles of Pten in modulating the naïve pluripotency maintenance of ESCs and to define the underlying molecular mechanism.

\section{Results}

Loss of tumor suppressor Pten maintains ESC pluripotency and modulates ESC differentiation

To explore the function of Pten in mouse ESCs, we generated $\mathrm{Pten}^{-/-}$ESCs using the CRISPR-Cas9 system (Fig. S1a). These cell lines contained mutant alleles with small insertions or deletions (indels) at the target sites. We utilized clone G1, in which the start codon of Pten was deleted in the genome to perform experiments (Fig. S1a). Pten deletion drove mouse ESCs toward a "domed" morphology (Fig. 1a), and Pten ${ }^{-1-}$ ESCs showed increased protein and mRNA levels of Nanog, Oct4, and Klf4 (Fig. 1b and S1b). Immunostaining with anti-Nanog and anti-Oct 4 antibodies confirmed that the expressions of Nanog and Oct4 proteins was upregulated in $\mathrm{Pten}^{-/-}$ ESCs (Fig. 1c). The elevated expression of pluripotency genes in $\mathrm{Pten}^{-/-}$ESCs prompted us to investigate the colony formation ability of these cells. Alkaline phosphatase (AP) staining for Pten ${ }^{-1-}$ and WT ESCs was used to examine their colony morphology (Fig. 1d). Compared with WT ESCs, a greater proportion of $\mathrm{Pten}^{-1-}$ ESCs exhibited "domed" morphologies (green arrows) (Fig. 1d, e). The effect of Pten deletion on the maintenance of ESC pluripotency might vary in different $\mathrm{Pten}^{-1-}$ cell lines, thus we compared three independent $\mathrm{Pten}^{-/-}$ESC lines with WT ESCs and found that all the Pten ${ }^{-1}$ ESC lines consistently showed a more pronounced ground state and higher expression of pluripotency genes than the WT ESCs (Fig. S1c, d). In addition, re-expression of Pten in $\mathrm{Pten}^{-/-}$ESCs by lentivirus system restored the expression of pluripotency genes (Fig. S1e). We performed transcriptomic analysis, and a pairwise comparison indicated that 242 genes were upregulated and 224 genes were downregulated in $\mathrm{Pten}^{-/}$ESCs (Fig. S1f). Multiple pluripotency markers were highly expressed in $\mathrm{Pten}^{-/-}$ ESCs (Fig. 1f). The principal component analysis (PCA) of gene expression revealed that Pten ${ }^{-/}$ESCs displayed distinct transcriptome profiles (Fig. S1g).

We also compared the expression of naïve and primed pluripotency genes between WT and Pten ${ }^{-1-}$ ESCs using heat map analysis and measured the mRNA levels of primed pluripotency genes (Otx2, Pou3f1, Lefty1, Lin28a, and Dnmt3b). Pten ${ }^{-1-}$ ESCs showed lower expression of the primed pluripotency genes and a higher expression of the naïve pluripotency markers (Fig. S2a, b). The protein level of the naïve marker Rex1 was also elevated in Pten $^{-1-}$ ESCs (Fig. S2c). Pten ${ }^{-1-}$ ESCs exhibited an augmented proliferation ability (Fig. S2d), which is consistent with the previous results of Hong Wu showing that loss of Pten enhanced cellular self-renewal capacity ${ }^{16,20}$. Propidium iodide (PI) incorporation assay and cell apoptosis assay indicated that Pten deletion slightly suppressed apoptosis in ESCs (Fig. S2e, f). Taken together, these results indicated that Pten deletion could maintain naïve pluripotency of ESCs.

The ESCs were then induced to form EBs to further verify the effect of Pten deletion on ESC differentiation. As expected, there were higher levels of Nanog and Oct4 in Pten $^{-1-}$ EBs than WT EBs, and the expression of Nanog and Oct 4 was decreased after 3 days and 5 days, respectively, in WT and $\mathrm{Pten}^{-1-}$ EBs (Fig. S3a). The expression of Nanog was turned off completely at 7 days in WT EBs but persisted for 9 days in Pten ${ }^{-/-}$EBs (Fig. S3a). In response to retinoic acid (RA), pluripotency gene expression is suppressed and ESCs undergo directed differentiation ${ }^{21}$. After treatment with RA for 3 days, the expression of Klf4 became undetectable in WT cells, while modest expression of Klf4 was retained in Pten ${ }^{-/-}$ cells, and the expression of Oct4 was higher in the Pten $^{-/-}$cells than WT cells (Fig. 1g). The mRNA levels of a series of differentiation markers decreased in Pten ${ }^{-/-}$ cells after RA treatment (Fig. S3b), and the protein levels of Mef2c (a mesoderm marker) and Gata4 (an endoderm marker) were reduced in $\mathrm{Pten}^{-/-}$cells (Fig. S3c), thus Pten deletion might delay the early differentiation in mouse ESCs. Pten ${ }^{-1-}$ cells displayed greater proliferation ability than WT cells (Fig. S3d), and Pten deletion slightly suppressed cell apoptosis after RA treatment (Fig. S3e). It is consistent with the conclusion of a previous report that PTEN induces cell apoptosis ${ }^{22}$. Meanwhile, both WT and Pten ${ }^{-/}$ESCs could differentiate into mesodermal, ectodermal, and endodermal tissues (Fig. S3f).

We established protocols for ectoderm, mesoderm, and endoderm differentiation in vitro, and the expression of Pax6 and Sox2 (ectodermal markers) significantly increased in the $\mathrm{Pten}^{-1-}$ cells at day 7, while the expression of Tubb3 (a neuron-specific marker) and Krt8 (a skin marker) slightly decreased (Fig. S3g). The result is consistent with a previous conclusion that knockdown of 


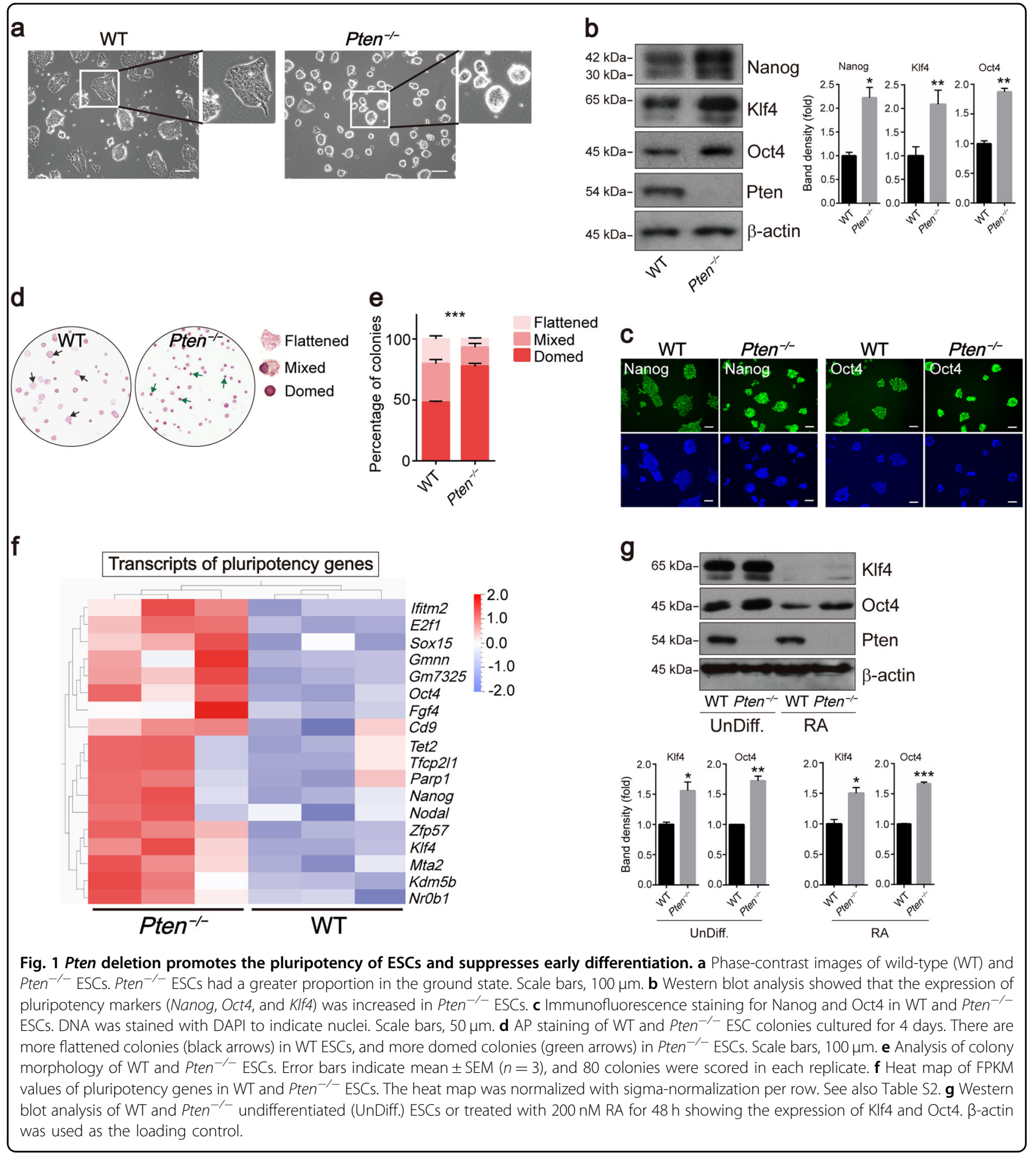

Pten in the mouse brain has been shown to increase the proliferation of neural progenitor cells but not result in a bias of neuron differentiation ${ }^{23}$. However, the expression of mesodermal markers (Mef2c, Kdr, MyoD1, and $M l c 2 v)$ and endodermal markers (Afp, Gata4, and FoxA2) declined (Fig. S3g). We detected a dynamic change of protein levels of Pax6, Mef2c, and Gata4 during differentiation from day 0 to day 9 . The protein level of Pax6 significantly increased from day 5, while the Mef2c and Gata4 levels were diminished (Fig. S3h). These results suggest that Pten deletion might delay the mesoderm and endoderm differentiation and that Pten regulates the early and later differentiation of ectodermal lineages through different mechanisms. 


\section{Pten deletion sustains ESC pluripotency by blocking Gsk3 $\beta$ activity}

ESCs established in the presence of $2 \mathrm{iL}$ are postulated to represent the ground state pluripotency ${ }^{24}$. Akt inhibits Gsk3 $\beta$ activity through the phosphorylation of Ser9 $9^{25,26}$, and a previous report mentioned that PTEN knockdown in human ESCs results in augmented self-renewal, survival, and proliferation but has no effects on GSK3 activity $^{15}$. In our results, WT and Pten ${ }^{-/-}$ESCs were cultured in medium including 2iL and in medium lacking PD0325901, CHIR99021, LIF, 2i, and 2iL, respectively (Fig. 2a). We found that Pten $^{-1-}$ ESCs still displayed a "domed" morphology in culture medium lacking CHIR99021 or LIF, while a large proportion of WT ESCs displayed a "flattened" morphology (Fig. 2a-c). The activity of the PI3K-Gsk3 $\beta$ pathway was examined when ESCs were cultured in medium without CHIR99021. The phosphorylation of Akt significantly increased in $\mathrm{Pten}^{-/-}$ ESCs, and the Gsk3 $\beta$ phosphorylation also increased, which is supposedly correlated with the inactivation of Gsk3 3 (Fig. 2d, S4d, and S4g). Besides, the protein expression levels of Nanog, Klf4, and Oct4 were increased in Pten ${ }^{-1-}$ ESCs in the absence of CHIR99021 (Fig. S4c). Sox2 is a substrate of Akt, and we measured the total level and phosphorylation level of Sox 2 in ESCs and EBs. Intriguingly, loss of Pten suppressed the expression of Sox2 in ESCs (Fig. S4e) but promoted the expression in EBs (Fig. S4f). In addition, the phosphorylation levels of Sox2 increased both in Pten ${ }^{-/}$ESCs and EBs when the phosphorylation levels were normalized to the total level of Sox2 (Fig. S4e, f). This indicated that loss of Pten activates Akt and that there might be a complex mechanism, through which Pten regulates the expression of Sox2 at different stages during ESCs differentiation.

Mouse ESCs are maintained by using the cytokine LIF to activate Stat3 signaling ${ }^{9-11}$, and PTEN negatively regulates PI3K/mammalian target of rapamycin (mTOR) $)^{27}$, which promotes STAT3 activity ${ }^{28}$. After the removal of LIF, the ESC maintenance by Pten deletion might be due to the activation of Stat3 (Fig. 2e). AKT lies at a signaling node downstream of PI3K, and the PI3K/AKT signaling pathway is sufficient to maintain the self-renewal and survival of stem cells ${ }^{29}$. We evaluated the effects of PI3K inhibitors (LY294002 and PX-866) on the pluripotency of $\mathrm{Pten}^{-/}$ESCs and found that PI3K inhibitors significantly abated the expression of pluripotency genes by inhibiting Akt activity (Fig. 2f). A previous result showed that PTEN deficiency augmented the levels of p-S6 for activating the $\mathrm{AKT} / \mathrm{mTOR}$ signaling pathway in human $\mathrm{ESCs}^{15}$. To identify alternative mechanisms involved in Ptenregulated ESC maintenance, we assessed the activity of several signaling pathways, including the MAPK and mTOR pathways, and loss of Pten promoted the mTOR signaling pathway (Fig. S5d). PI3K/Akt/mTOR is known to play key roles in cell adhesion, proliferation, and survival. In the PI3K/Akt/mTOR pathway, Pten acts as a brake upstream of Akt, and Akt activates mTOR via phosphorylation of TSC2, and this regulation mechanism was revealed in many biological processes ${ }^{30,31}$. mTOR inhibition by rapamycin could rescue the expression of Klf4 and Nanog in Pten ${ }^{-1-}$ ESCs (Fig. S5e). Our results indicate that PI3K/Akt/mTOR might be another signaling pathway regulated via Pten in modulating ESCs' naïve pluripotency.

$\beta$-catenin is a downstream factor of Gsk3 $\beta$, and Wnt/ $\beta$-catenin signaling plays important roles in ESC selfrenewal ${ }^{32,33}$. In our results, the cytoplasm $/$ membrane distribution of $\beta$-catenin was reduced in $\mathrm{Pten}^{-/}$ESCs, while a higher nuclear abundance of $\beta$-catenin was observed (Fig. 2g). The immunofluorescence result showed that $\beta$-catenin was enriched in the membrane of WT ESCs (Fig. 2h). We also verified that the mRNA levels of Wnt-target genes such as $c-M y c$, Jun, Dkk1, Axin2, and Sp5 increased (Fig. S4i) and that the protein levels of cMyc and Jun also increased in Pten ${ }^{-1-}$ ESCs (Fig. S4j). We supposed that inhibition of Gsk3 $\beta$ in Pten ${ }^{-1-}$ ESCs might stabilize $\beta$-catenin and that the stabilized $\beta$-catenin translocated to the nucleus to mimic stimulation of canonical Wnt signaling, which restricts the conversion from naïve to primed pluripotency ${ }^{34}$.

ESCs were cultured in medium with different concentrations of CHIR99021 or LIF (Fig. S4a and S5a). CHIR99021 is utilized to maintain naïve pluripotency in mouse $\mathrm{ESCs}^{35}$. An increased proportion of WT ESCs exhibited "domed" morphology as the concentration of CHIR99021 increased, and the proportion of groundstate WT ESCs was equivalent to Pten ${ }^{-/}$ESCs at $4 \mu \mathrm{M}$ and $5 \mu \mathrm{M}$ CHIR99021 (Fig. S4a, b). The protein level of pluripotency markers in WT ESCs was also similar to Pten $^{-/-}$ESCs at $4 \mu \mathrm{M}$ and $5 \mu \mathrm{M}$ CHIR99021 (Fig. S4h). These results indicated that $\mathrm{Pten}^{-1-}$ ESCs might sustain ground pluripotency with lower concentrations of CHIR99021 than those needed by WT ESCs, supporting the hypothesis that Gsk3 $\beta$ kinase is more inhibited in Pten $^{-/-}$ESCs. It is consistent with the previous data that Pten $^{-1-}$ ESCs showed higher phosphorylation levels of Gsk3 $\beta$ which is supposedly correlated with the inactivation of Gsk3 $\beta$ (Fig. $2 \mathrm{~d}$ ). In addition, LIF could not revert WT ESCs to a similar proportion of domed colonies as Pten ${ }^{-1-}$ ESCs (Fig. S5a, b). Fig. S5c showed that the loss of Pten had no effect on the activity of Stat3 when ESCs were cultured in medium with 2iL. This suggests that LIF/Stat3 signaling might be not the main signaling pathway regulated by Pten in modulating ESC pluripotency.

These results indicated that $\mathrm{Pten}^{-/-}$ESCs maintain pluripotency primarily through inhibiting Gsk $3 \beta$, similar to the role of the Gsk3 $\beta$-inhibitor CHIR99021. 
a

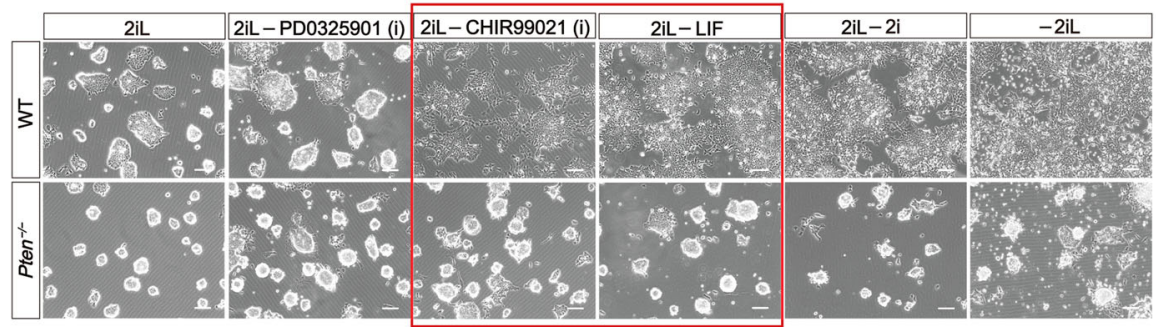

b

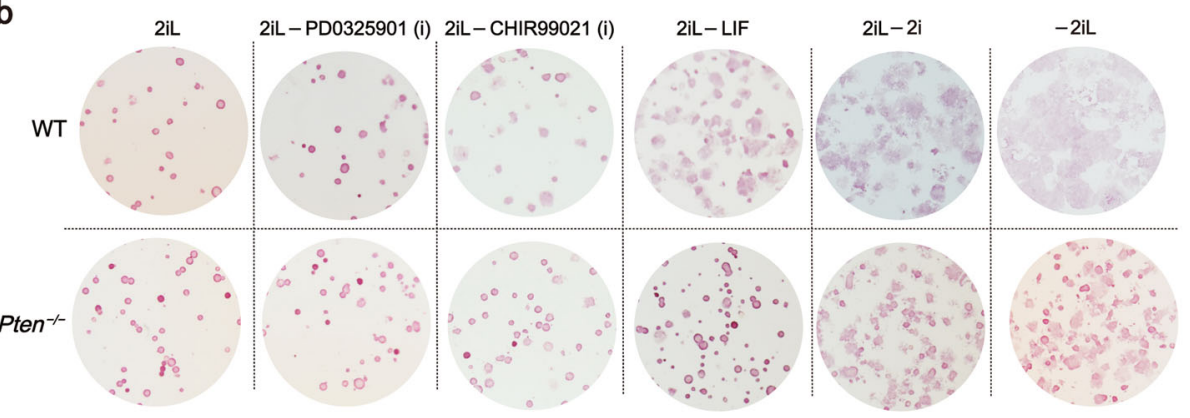

C
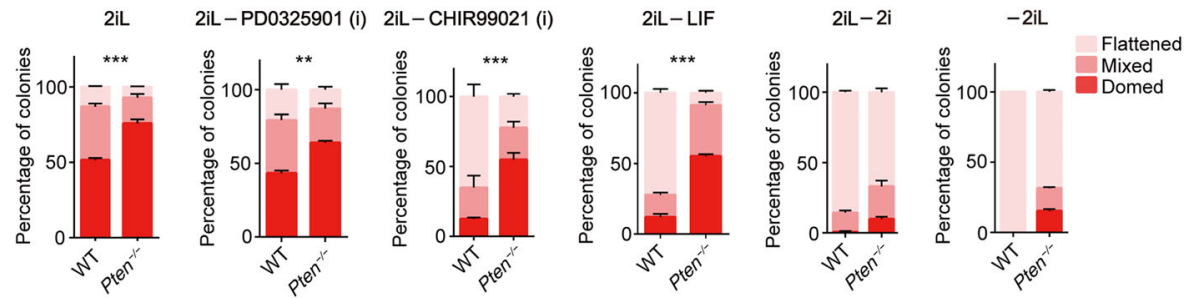

d

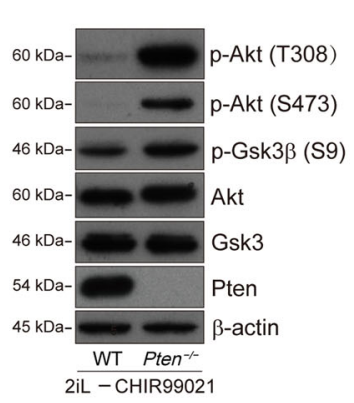

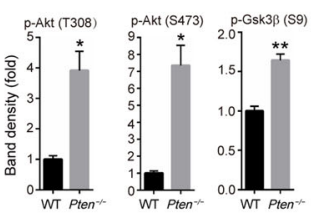

g

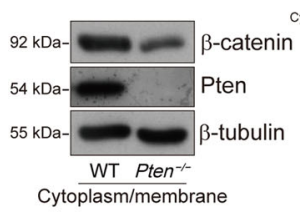

Cytoplasm/membrane
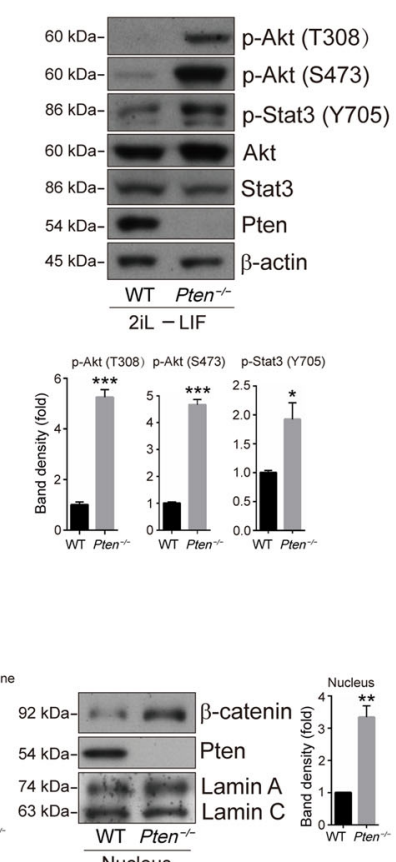

e

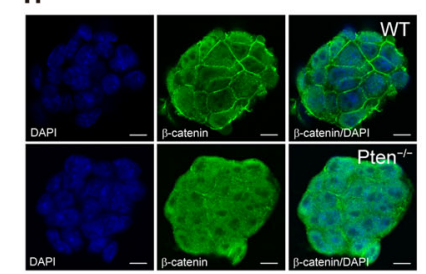

Fig. 2 (See legend on next page.) 
(see figure on previous page)

Fig. 2 Pten deletion maintains ESCs by regulating the PI3K-Gsk3 $\beta$ signaling axis. a Phase-contrast images of WT and Pten ${ }^{-1-}$ ESCs cultured in medium including 2iL and in medium lacking PD0325901, CHIR99021, LIF, 2i, and 2iL, respectively. Scale bars, $100 \mu$ m. b, c Analysis of AP staining of WT and Pten ${ }^{-1}$ ESC colonies cultured in medium including 2iL and in medium lacking PD0325901, CHIR99021, LIF, 2i, and 2iL, respectively. Without CHIR99021 and LIF, respectively, Pten ${ }^{-1-}$ ESC colonies displayed more than 50\% domed morphology, but WT ESC colonies showed a large reduction in domed morphology. Error bars indicate mean $\pm \operatorname{SEM}(n=3)$, and 80 colonies were scored in each replicate. $\mathbf{d}$ Western blot analysis of WT and Pten $^{-1-}$ ESCs cultured in medium without CHIR99021 showing the phosphorylation of Akt at T308 and S473 and the phosphorylation of Gsk3ß at S9. e Western blot analysis of WT and Pten ${ }^{-1-}$ ESCS cultured in medium without LIF showing the phosphorylation of Akt at T308 and S473 and the phosphorylation of Stat3 at Y705. $\mathbf{f}$ Western blot analysis of Pten ${ }^{-1}$ ESCs treated with PI3K inhibitors LY294002 and PX-866 showing the expression of the pluripotency markers and the phosphorylation of Akt at T308 and S473. g Protein levels of $\beta$-catenin in the cytoplasm/membrane and nucleus of WT and Pten ${ }^{-1-}$ ESCs. $\mathbf{h}$ Immunofluorescence staining for $\beta$-catenin in WT and Pten ${ }^{-1-}$ ESCs. DNA was stained with DAPI to indicate nuclei. Scale bars, $10 \mu \mathrm{m}$.

The Pten-inhibitor SF1670 contributes to ESC maintenance

PI3K/Akt signaling is crucial for stem cell self-renewal, and the active form of Akt sustains pluripotency in mouse and primate $\mathrm{ESCs}^{36,37}$. We hypothesized that the activation of the PI3K/Akt pathway by small-molecule inhibitors of Pten maintains the pluripotency of ESCs. We treated mouse ESCs with several Pten inhibitors (data not shown) and found that the Pten-specific inhibitor SF1670 maintained the pluripotency of ESCs (Figs. 3a, b, and S6a). SF1670 maintained ESC pluripotency as indicated by the increased proportion of colonies with a "domed" morphology (green arrows), while DMSO-treated ESCs showed a higher proportion of colonies with a "flattened" morphology (black arrows) (Fig. 3c, d). The transcript levels of several pluripotency genes were significantly increased by SF1670 (Fig. 3e). The protein levels of pluripotency genes were also elevated in ESCs treated with SF1670 (Fig. 3f and S6e). SF1670 is a specific inhibitor of Pten, and as expected, the SF1670-treated WT ESCs exhibited similar "domed" morphologies as the Pten $^{-1-}$ ESCs (Fig. 3g, h, i). We also evaluated the teratoma formation ability and found that the weight of teratomas derived from both SF1670-treated WT ESCs and Pten $^{-1-}$ ESCs increased compared with those derived from WT ESCs (Fig. 3j, k). Fig. S6b shows the colony morphology of WT ESCs, WT ESCs treated with different concentrations of SF1670, and Pten ${ }^{-1-}$ ESCs cultured in ESC culture medium. The proportion of cells with "domed" morphologies rose as the SF1670 concentration increased (Fig. S6c, d) indicating that the Pten-inhibitor SF1670 contributed to ESC maintenance.

\section{The Pten-inhibitor SF1670 plays a similar role to the Gsk3ß-inhibitor CHIR99021 in ESC maintenance}

SF1670 is a small-molecule inhibitor of Pten and can increase the $\mathrm{PIP}_{3}$ level in transfused neutrophils ${ }^{38}$. The DMSO-treated and SF1670-treated WT ESCs were cultured in medium including $2 \mathrm{iL}$ and in medium lacking PD0325901, CHIR99021, LIF, 2i, and 2iL, respectively, and SF1670 sustained the "domed" morphology of WT ESCs in the absence of CHIR99021 (Fig. S7a, b), while LIF could not be substituted by SF1670 for inadequate activation of Stat3 (Fig. S7a, b, e). SF1670 at $2 \mu \mathrm{M}$ also eliminated the loss of the "domed" morphology of ESCs seen in the absence of CHIR99021 (Figs. 4a, and S7c). We also measured the transcript levels of pluripotency genes (Esrrb, Zfp42, and Fgf4) in WT ESCs at different time points in the absence of CHIR99021. Their expression levels were higher in SF1670-treated ESCs than in DMSO-treated ESCs (Fig. 4b). Immunostaining result confirmed that the expression of Nanog protein was upregulated in SF1670-treated ESCs (Fig. S7d). The phosphorylation levels of Akt at T308 and S473 and Gsk3 $\beta$ at S9 were significantly augmented in SF1670treated ESCs cultured in medium without CHIR99021 (Fig. 4c). The protein level of the naïve marker Rex1 was slightly elevated in SF1670-treated ESCs (Fig. S7f), and the proportion of Rex1 positive cells was also slightly increased in SF1670-treated ESCs compared to DMSOtreated ESCs (Fig. S7g). In addition, the in vivo teratoma formation ability was examined, and the teratomas derived from SF1670-treated ESCs were larger than those formed from DMSO-treated ESCs (Fig. 4d, e). Meanwhile, the DMSO-treated and SF1670-treated ESCs could differentiate into mesodermal, ectodermal, and endodermal tissues (Fig. 4f). On the other hand, the cell proliferation ability of SF1670-treated ESCs increased compared with DMSO-treated ESCs (Fig. S7h), and SF1670 slightly suppressed the apoptosis of ESCs (Fig. S7i).

These results indicate that Pten inhibition by SF1670 plays a similar role to the Gsk3 $\beta$-inhibitor CHIR99021 in ESC maintenance.

\section{Pten activation by S380A, T382A, and T383A (Pten-A3) mutations suppresses ESC pluripotency}

Phosphorylation of the C-terminal of PTEN at S380, T382, and T383 can inhibit its phosphatase activity and favor its stabilization ${ }^{39,40}$. We have shown that Pten inhibition or deletion sustained the ESC pluripotency (Figs. 1-4). To explore the underlying molecular mechanism, we hypothesized that the pluripotency of ESCs would be influenced when Pten was activated by 


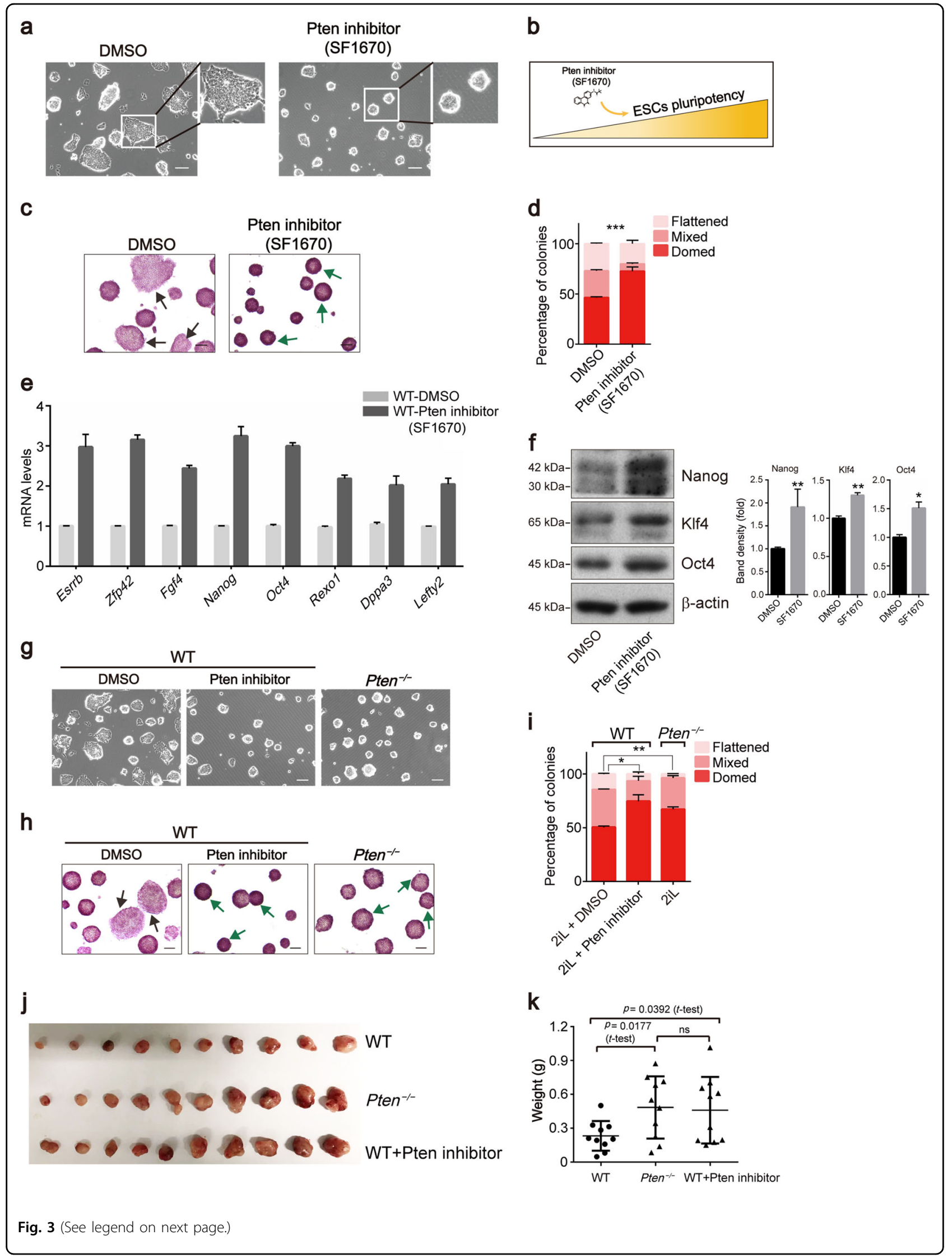


(see figure on previous page)

Fig. 3 The Pten-inhibitor SF1670 contributes to sustaining ESC pluripotency. a Phase-contrast images of WT ESCs treated with DMSO and the Pten-inhibitor SF1670 when cultured in medium with 2i and LIF (2iL). Scale bars, $100 \mu \mathrm{m}$. b Pten-inhibitor SF1670 promotes the ESC pluripotency. c Alkaline phosphatase staining of WT ESC colonies treated with DMSO and SF1670. There are more flattened colonies (black arrow) in DMSO-treated ESCs, and more domed colonies (green arrow) in SF1670-treated ESCs. d Analysis of colony morphology of WT ESCs treated with DMSO and SF1670. Error bars indicate mean $\pm \operatorname{SEM}(n=3)$, and 80 colonies were scored in each replicate. e qRT-PCR analysis of mRNA expression of pluripotency markers (Esrrb, Zfp42, Fgf4, Nanog, Oct4, Rexo 1, Dppa3, and Lefty2) in DMSO-treated ESCs and SF1670-treated ESCs. Error bars indicate mean \pm SD $(n=3)$. f Western blot analysis of WT ESCS treated with DMSO and SF1670 showing the expression of the pluripotency markers Nanog, KIf4, and Oct4. g Phase-contrast images of DMSO-treated WT, SF1670-treated WT, and Pten ${ }^{-1-}$ ESCs when cultured in medium with $2 \mathrm{iL}$. Scale bars, $100 \mu \mathrm{m} . \mathbf{h}$ AP staining of DMSO-treated WT, SF1670-treated WT, and Pten ${ }^{-1-}$ ESC colonies when cultured in medium with 2iL. i Analysis of colony morphology of DMSO-treated WT, SF1670-treated WT, and Pten ${ }^{-1-}$ ESC colonies. Error bars indicate mean \pm SEM $(n=3)$, and 80 colonies were scored in each replicate. $\mathbf{j}$ Nude mice were injected with WT ESCs, Pten ${ }^{-1}$ ESCs, and SF1670-treated WT ESCs on the left and right sides, respectively. Teratomas were generated at the contralateral positions aligned at the same positions in each treatment. $\mathbf{k}$ Weights (g) of teratomas from WT ESCs, Pten ${ }^{-1-}$ ESCs, and SF1670-treated WT ESCS.

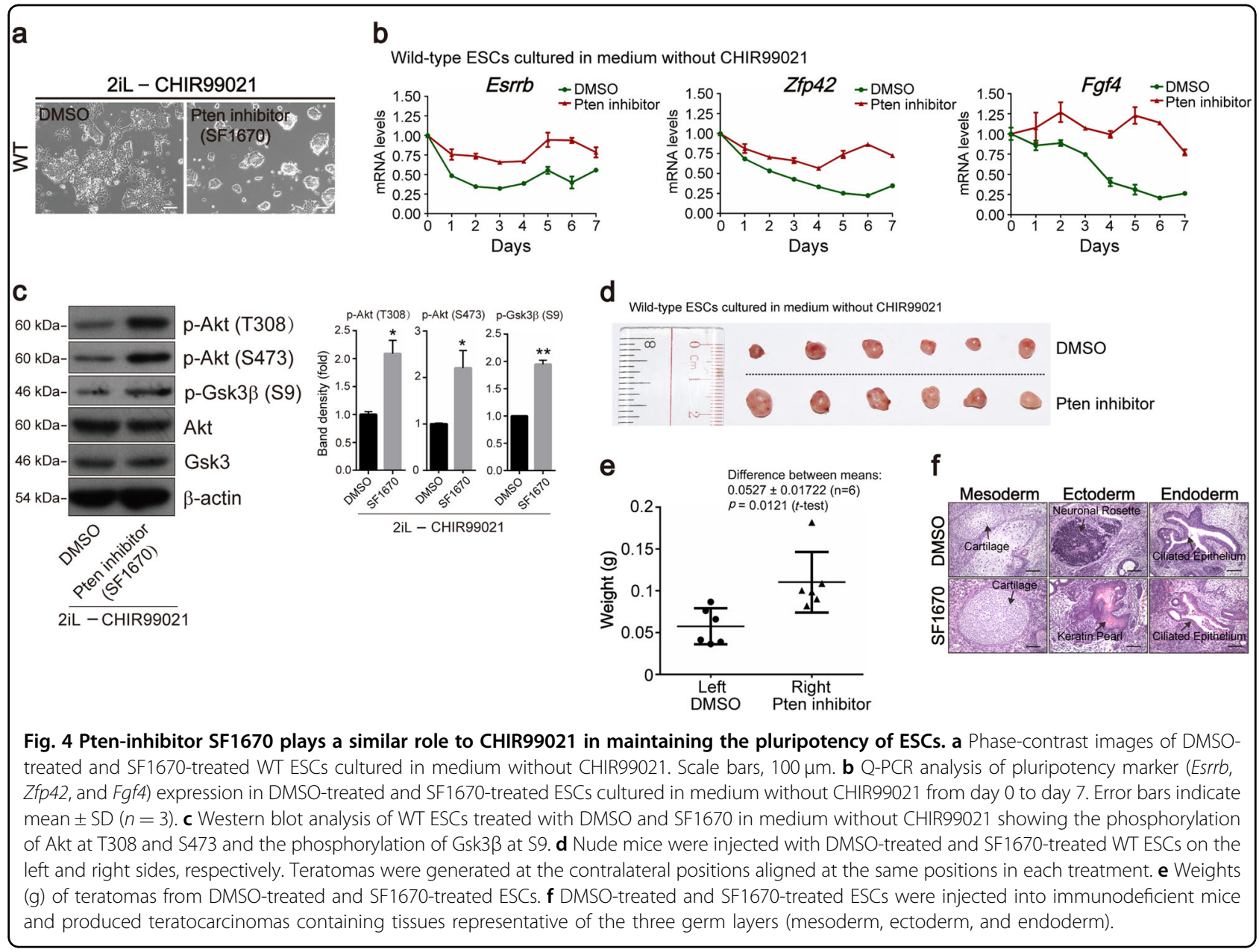

S380A, T382A, and T383A (Pten-A3) mutations that prevent their phosphorylation (Fig. 5a, b). Pten-A3 mutant ESCs were generated by using the CRISPR-Cas9 system (Fig. S8a). Phosphorylation of S380, T382, and T383 could not be detected in Pten-A3 mutant ESCs by Western blot (Fig. 5c and S8b). The protein levels of Oct4 and Klf4 were decreased in Pten-A3 mutant ESCs but were significantly increased in Pten ${ }^{-/-}$ESCs (Fig. 5d). We also measured the expression of pluripotency genes in two other Pten-A3 mutant cell lines, and the result was consistent with Fig. 5d (Fig. S8b). Overexpression of Pten in Pten-A3 mutant ESCs could restore the expression of pluripotency genes (Fig. S8c). We stained the ESC colonies with an anti-Nanog antibody, and the signal in the 


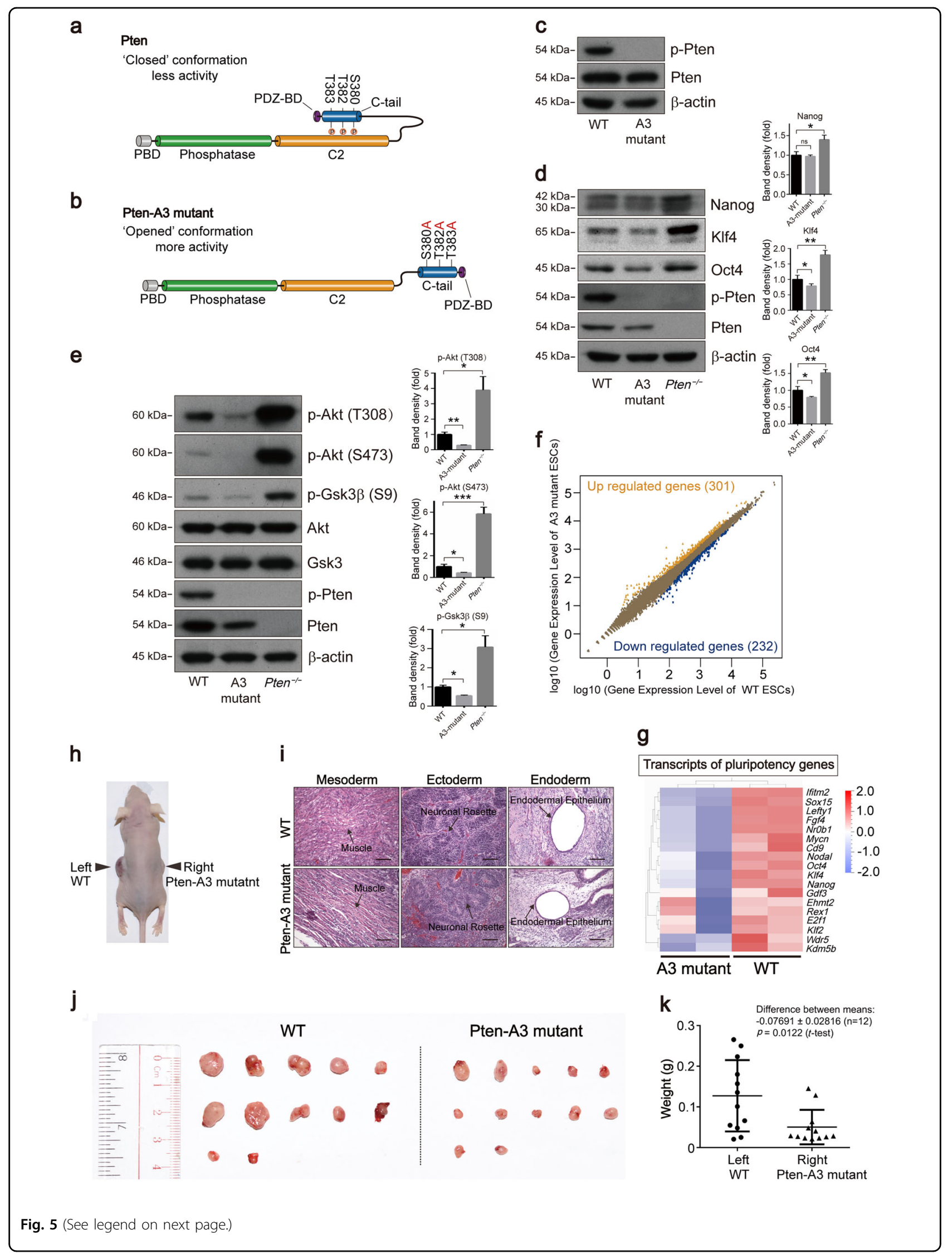


(see figure on previous page)

Fig. 5 Pten activation by S380A, T382A, T383A mutations suppresses ESC pluripotency. $\mathbf{a}, \mathbf{b}$ The domain structure of Pten in the "closed" conformation and "opened" conformation. c Western blots analysis showing the loss of phosphorylation in the Pten-A3 mutant ESCs. $\mathbf{d}$ Western blot analysis of WT, Pten-A3 mutant, and Pten ${ }^{-1-}$ ESCs showing the expression of the pluripotency markers Nanog, Klf4, and Oct4. e Phosphorylation of Akt at $\mathbf{S 4 7 3}$ and T308 and phosphorylation of Gsk3 3 at S9 by Western blot. $\mathbf{f}$ Scatter plots of transcript expression in WT and Pten-A3 mutant ESCs. Expression values are shown on a log 10 scale. Yellow dots indicate upregulated genes in Pten ${ }^{-1-}$ ESCs, and blue dots indicate downregulated genes (fold-change cutoff $=1.5$, FDR threshold $\leq 0.05$ ). $\mathbf{g}$ Heat map of FPKM values of pluripotency genes in WT and Pten-A3 mutant ESCs. The heat map was normalized with sigma-normalization per row. See also Table S3. $\mathbf{h}$ A nude mouse injected with WT and Pten-A3 mutant ESCs on the left and right sides, respectively. i WT and Pten-A3 mutant ESCs were injected into immunodeficient mice and produced teratocarcinomas containing tissues representative of the three germ layers (mesoderm, ectoderm, and endoderm). $\mathbf{j}$ Teratomas from WT and Pten-A3 mutant cells generated at the contralateral positions aligned at the same positions in each genotype. $\mathbf{k}$ Weights ( $\mathrm{g}$ ) of teratomas from WT and Pten-A3 mutant cells.

Pten-A3 mutant ESCs was weaker than in the WT ESCs (Fig. S8d). Furthermore, AP staining showed that the proportion of domed colonies was decreased in the PtenA3 mutant ESCs (Fig. S8e, f, g), and the proliferation ability of Pten-A3 mutant ESCs was also inhibited (Fig. S8h).

The experiments described above showed that the PI3K-Gsk3 $\beta$ pathway is involved in regulating the ESC pluripotent state via Pten. The phosphorylation of Akt and Gsk3 $\beta$ was significantly decreased in the Pten-A3 mutant ESCs compared with WT ESCs but was significantly increased in the Pten ${ }^{-1-}$ ESCs (Fig. 5e). The stability and activity of Pten are linked to the phosphorylation of the S380, T382, and T383 residues in the Cterminal tail ${ }^{41}$. Pten activity was inhibited, but the expression of Pten also decreased in the Pten-A3 mutant ESCs (Fig. 5d, e). Thus, there was a balance for Pten-3A mutant ESCs in the regulation of ESC pluripotency between inhibition of Pten activity and the degradation of Pten.

Transcriptome analysis of WT and Pten-A3 mutant ESCs was performed, and a scatter plot analysis showed the difference between the WT and Pten-A3 mutant ESCs (Fig. 5f). The reduced expression of pluripotency markers in Pten-A3 mutant ESCs was shown in the heat map (Fig. 5g). We then examined the teratoma formation ability of Pten-A3 mutant ESCs in vivo. The WT and Pten-A3 mutant ESCs were injected into immunodeficient mice and both gave rise to multidifferentiated teratomas that contained mesodermal, ectodermal, and endodermal tissues (Fig. 5h, i), while the teratomas derived from Pten-A3 mutant cells were significantly smaller than those derived from WT ESCs (Fig. 5j, k).

Our results indicate that phosphorylation levels of Pten at S380, T382, and T383 are important for Pten to regulate the ESC pluripotent state by inhibiting the activity of Pten and promoting Akt activity.

\section{Suppression of Pten regulates lineage choice and differentiation during embryoid body formation}

Pten deletion or inhibition sustained ESC pluripotency, suggesting that Pten plays an important role during early embryogenesis. We measured the expression of the three embryonic germ layer markers by PCR. The expression of the ectoderm markers was significantly upregulated in Pten $^{-1-}$ EBs and the endoderm markers were downregulated (Fig. 6a).

We used RNA-Seq to compare the transcriptomes of EBs derived from $\mathrm{Pten}^{-1-}$ and WT ESCs. A pairwise comparison indicated that 435 genes were significantly upregulated in $\mathrm{Pten}^{-/-}$EBs, including ectoderm genes (Ascl1, Sox1, Pax6, and Prom1) and pluripotency genes (Nanog, Oct4, Esrrb1, Sox2, and Zfp42), and 435 genes were significantly downregulated, including the endoderm gene Gata6 (Fig. 6b). PCA and Pearson correlation coefficients for all gene expression showed distinct expression patterns in WT and Pten ${ }^{-1-}$ EBs (Fig. S9a, b). The expression of the lineage-specific markers in WT and ten $^{-/-}$EBs was visualized in the heat map, and ectoderm and pluripotency markers were increased in $\mathrm{Pten}^{-1-}$ EBs while endoderm markers were decreased (Fig. 6c). We measured the expression of the representative lineage-specific markers by qRT-PCR, and the expressions of the ectoderm and pluripotency markers were elevated in Pten $^{-1-}$ EBs compared with WT EBs, but endoderm markers were decreased (Fig. 6d). In addition, the expression patterns of the ectoderm and pluripotency markers in WT EBs treated with $2 \mu \mathrm{M}$ SF1670 were consistent with the results in Pten ${ }^{-/-}$EBs (Fig. 6d, e). The expression levels of ectoderm markers Pax6 and Sox 1 were measured on different days, and there was a significant difference between WT and Pten ${ }^{-/-}$EBs after culturing for 8 days (Fig. 6f). Fig. S9c shows the expression of ectoderm markers (Sox1, Sox3, Brn2, Ascl1, Nestin, Msi1, Prom1, and Pax6) for three WT and three Pten ${ }^{-/-}$EBs.

These results suggest that Pten deletion or Pten inhibition promotes ectoderm differentiation, inhibits endoderm differentiation, and maintains the pluripotency of mouse ESCs.

\section{Discussion}

Mouse and human ESC lines are derived from blastocysts and provide important models to investigate early embryogenesis ${ }^{1,2}$. ESCs can be maintained by some small- 


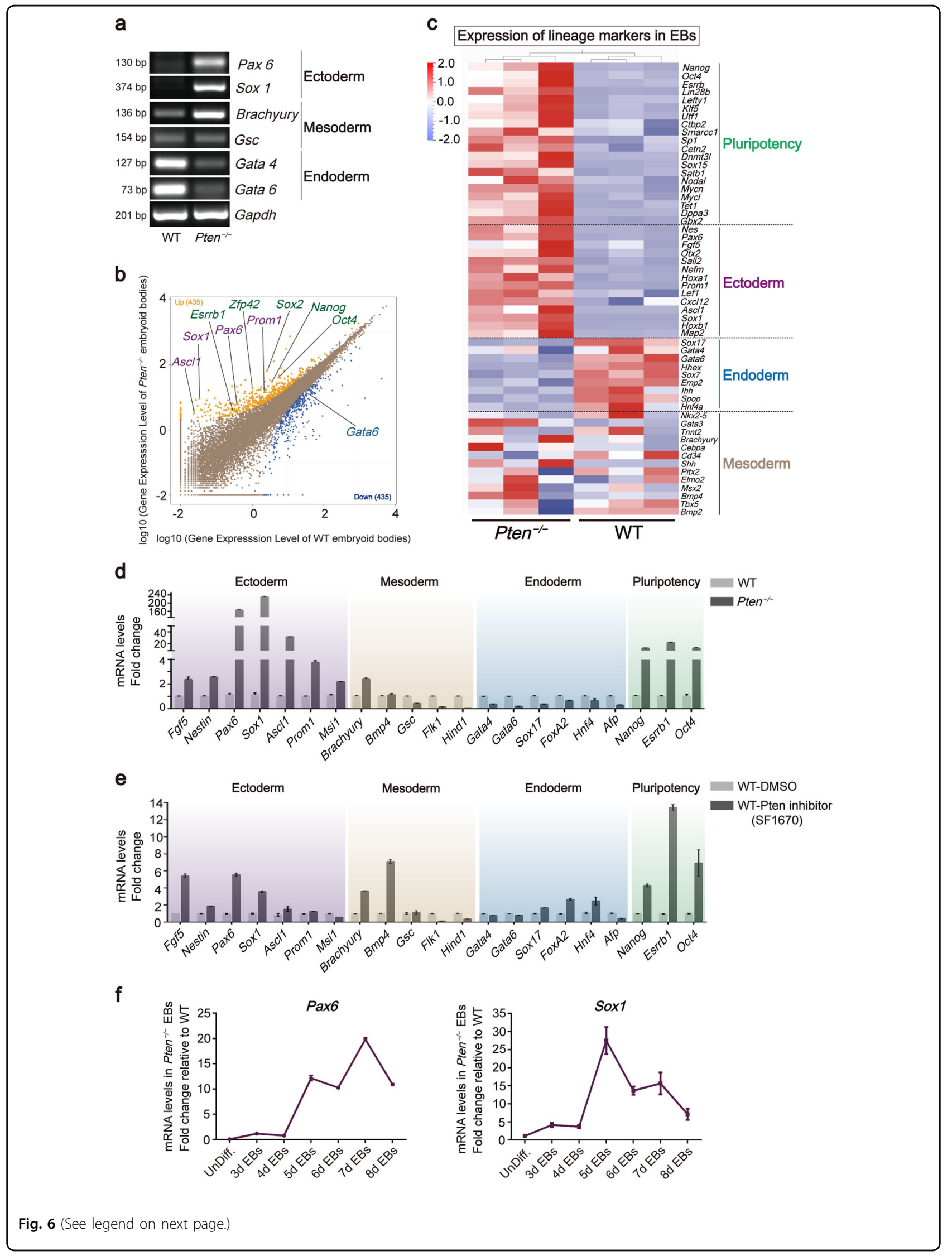


(see figure on previous page)

Fig. 6 Pten deletion and Pten inhibition regulate lineage choice and differentiation in mouse EBs. a RT-PCR analysis of EBs derived from WT and Pten $^{-1-}$ ESCs showing the expression of lineage-specific markers. b Scatter plot of transcript expression in WT and Pten ${ }^{-1-}$ EBs. Expression values are shown on a $\log 10$ scale. Yellow dots indicate upregulated genes in Pten ${ }^{-1-}$ EBs, and blue dots indicate downregulated genes. Probability $\geq 0.8$ and abs $(\log 2(Y / X)) \geq 1$. c Heat map of FPKM values of pluripotency, ectoderm, endoderm, and mesoderm markers in WT and Pten ${ }^{-1-}$ EBs. The heat map was normalized with sigma-normalization per row. See also Table S4. $\mathbf{d}$ The expression of lineage-specific markers was assessed by qRT-PCR in WT and Pten ${ }^{-/-}$EBs. Error bars indicate mean \pm SD $(n=3)$. e The expression of lineage-specific markers was assessed by qRT-PCR in WT EBs treated with DMSO or SF1670. Error bars indicate mean \pm SD $(n=3)$. f Expression of ectoderm markers Pax6 and Sox 1 in WT and Pten ${ }^{-1-}$ EBs at different days. Error bars indicate mean \pm SD $(n=3)$.

molecule inhibitors such as Gsk3 $\beta$-specific inhibitors and MAPK inhibitors. Here, our results showed that Pten deletion and the Pten-inhibitor SF1670 sustained ESC pluripotency by inhibiting Gsk3 $\beta$ (Fig. 7). This study thus demonstrates the functions of Pten in modulating the naïve pluripotency of ESCs and differentiation and has identified the regulatory mechanism.

Inhibition of Pten and p53 can drive increased Myc protein levels and promote an undifferentiated state with high renewal potential in mouse neural stem cells ${ }^{13}$. This is also an indication that Pten can regulate stem cell differentiation. We calculated the proportion of suspended and attached EBs of WT and Pten ${ }^{-1-}$ EBs. Intriguingly, fewer of $\mathrm{Pten}^{-/-}$EBs were attached on the Petri dishes (Fig. S3i). Lineage-specific differentiation is influenced by pluripotency states, for example, naïve versus primed states of pluripotent stem cells are not functionally equipotent in lineage-specific differentiation, and naïve pluripotent stem cells possess impaired mesoderm and endoderm capacity but enhance the capacity for neural differentiation ${ }^{42}$. In our results, Pten ablation promoted mouse ESCs to a more naïve pluripotency (Fig. S2a, b), and $\mathrm{Pten}^{-/-}$ESCs displayed an augmented differentiation ability for neural progenitor cells and delayed the mesoderm and endoderm differentiation (Fig. S3g, h). The effects of Pten deletion on lineage differentiation might be due to the naïve state.

Gene Ontology analysis by using DAVID functional innovation was performed with the RNA-seq data of WT and $\mathrm{Pten}^{-/}$EBs, WT and Pten ${ }^{-/}$ESCs, and WT and Pten-A3 mutant ESCs and showed that the PI3K/Akt pathway was enriched in these cell lines (Fig. S9d, e, f). Different signaling pathways were enriched in various cell lines, for example, Ras signaling pathway and Rap1 signaling pathway were enriched in wild-type and Pten $^{-1-}$ EBs (Fig. S9d), and Hippo signaling pathway, Calcium signaling pathway, and cAMP signaling pathway were enriched in wild-type and Pten-A3 mutant ESCs (Fig. S9f). The enrichment of signaling pathways in different cell lines suggests that there may exist a complex and dynamic signaling network at different stages of embryonic development. According to our current results, we supposed that Pten modulated ESC pluripotency primarily through regulating PI3K/Akt/Gsk3 $\beta$ signaling pathway.

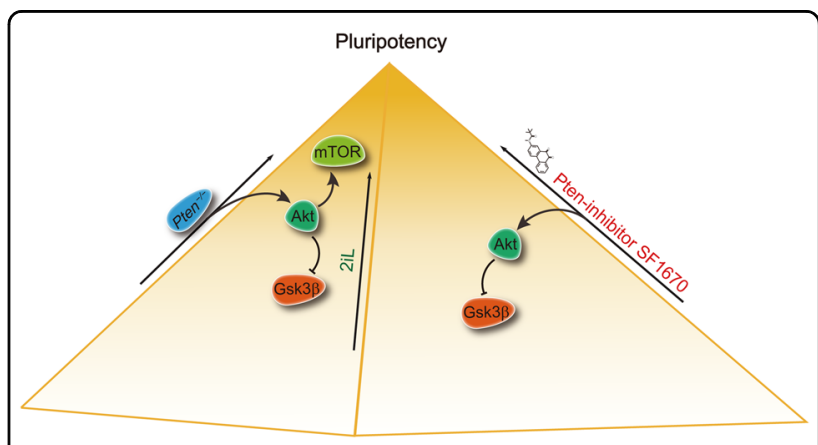

Fig. 7 A model for how Pten deletion or inhibition promotes ESC pluripotency. Pten deletion or inhibition promotes naïve pluripotency of mouse ESCs by activating Akt and further suppressing Gsk3ß. In addition, the Pten/Akt/mTOR signaling pathway is also involved in regulating ESC maintenance.

Our data showed that inhibition of Pten maintained ESCs pluripotency, and we defined the underlying molecular mechanism. This supports previous work showing that inhibition of Pten promotes the generation of induced pluripotent stem cells from mouse embryonic fibroblasts $^{43}$. We hypothesize that Pten inhibition is potentially useful in mouse ESC culture and regenerative medicine because of its role in ESC maintenance.

\section{Materials and methods \\ Animals}

All mouse procedures were performed in accordance with the recommendations of the Animal Experimentation Ethics Committee of The Chinese University of Hong Kong. The protocol was approved by the Animal Experimentation Ethics Committee of the Chinese University of Hong Kong (Ref No. 17-206-MIS).

\section{Mouse ESC culture}

The mouse E14 cell line was purchased from ATCC (ES-D3, ATCC, CRL-1934). Mouse ESCs were cultured in DMEM/F12 containing GlutaMAX and sodium pyruvate (Life Technologies, 10565) with 15\% fetal bovine serum (Hyclone, SH30071), nonessential amino acid solution (Life Technologies, 11140), and $\beta$-mercaptoethanol (Life Technologies, 31350) with $1 \times 10^{3}$ units/ml LIF (Millipore, ESG1106), $1 \mu \mathrm{M}$ PD0325901 (Sigma, PZ0162), and 
$2.5 \mu \mathrm{M}$ CHIR99021 (Sigma, SML1046). The cell line was tested for mycoplasma contamination.

\section{Pten deletion and Pten-A3 mutations in mouse ESCs by CRISPR-Cas9 technologies}

Guide sequences of sgRNA were incorporated into the pSpCas9(BB)-2A-GFP vector (Addgene, 48138) containing Cas9 and green fluorescent protein (GFP) genes. ESCs were transfected with constructs and then subjected to cell sorting of GFP-positive cells after transfection for $48 \mathrm{~h}$ (Figs. S1a and S8a). Finally, the Pten ${ }^{-/-}$and Pten-A3 mutant cell lines were identified by sequencing, and the cells were stored in liquid nitrogen. The sgRNA sequence and sequences of the primers are shown in Table S1.

\section{Colony-formation assay}

For the colony formation assay, ESCs were cultured in gelatin-coated plates at 250 cells per $\mathrm{cm}^{2}$ for 4 days. AP activity was detected by using an AP Staining Kit II according to the product manual (STEMGENT, 00-0055). Sample size was determined based on previous experience. At least three times of biological replicates have been performed for each cell line, and 80 colonies were scored in each replicate. The person collecting outcome measures and analyzing data was blinded to group allocation.

\section{Teratoma formation and histological analysis}

ESCs were dissociated and suspended in phosphatebuffered saline (PBS) supplemented with 50\% Matrigel (BD, 354234) at $1 \times 10^{6}$ cells $/ \mathrm{ml}$. A total of $5 \times 10^{4}$ cells were injected subcutaneously into recipient nude mice. To minimize the inter-individual variability in teratoma growth, two groups of ESCs were injected in the left and right side, respectively, in the same individuals. Sample size was determined based on previous experience and chosen based on putative effects sizes in pilot experiments. Animals were allocated to injection groups with different cell lines by randomization, and the person performing animal injection was blinded the sample groups. Cells (lines WT ESCs, Pten ${ }^{-/-}$ESCs, and SF1670treated WT ESCs) were injected into nude mice (ten mice per line); cells (lines DMSO-treated WT ESCs and SF1670-treated WT ESCs) were injected into nude mice (six mice per line); and cells (lines WT ESCs and Pten-A3 mutant ESCs) were injected into nude mice (12 mice per line). Three weeks later, the teratomas were isolated, fixed with $4 \%$ paraformaldehyde (PFA) in PBS, and stained with hematoxylin and eosin.

\section{Immunofluorescence}

After culturing for 3 days, ESCs were fixed with 4\% PFA for $20 \mathrm{~min}$ and incubated in PBS with $0.3 \%$ Triton-X 100 for $10 \mathrm{~min}$. After blocking with $5 \%$ bovine serum albumin in PBS, ESCs were stained with primary antibodies and incubated overnight at $4{ }^{\circ} \mathrm{C}$. The next day, samples were incubated with donkey anti-rabbit IgG highly crossadsorbed secondary antibodies (Life Technologies, A16036) for $2 \mathrm{~h}$ at RT. The following antibodies were used for immunofluorescence analysis: anti-Nanog (Cell Signaling Technology, 8822), anti- $\beta$-catenin (Cell Signaling Technology, 8480), and anti-Oct4 (Cell Signaling Technology, 2840).

\section{Western blot}

Cells were scraped from culture plates and incubated for $30 \mathrm{~min}$ in ice-cold lysis buffer containing protease inhibitor cocktails. For nuclear and cytoplasmic fractionation, the cells were collected by trypsin digestion and incubated in the cytoplasmic lysis buffer $(10 \mathrm{mM}$ Hepes, $1.5 \mathrm{mM} \mathrm{MgCl}_{2}, 10 \mathrm{mM} \mathrm{KCl}, 0.5 \mathrm{mM}$ DTT, $300 \mathrm{mM}$ sucrose, $0.1 \%$ NP-40, and protease inhibitors cocktail). Cells were lysed for $10 \mathrm{~min}$ on ice and spun for $15 \mathrm{~s}$ at $12,000 \mathrm{rpm}$ to collect the cytosolic lysate. Pellets were washed two times with cytoplasmic lysis buffer and lysed with nuclear lysis buffer $(50 \mathrm{mM}$ Hepes, $250 \mathrm{mM} \mathrm{KCl}$, $0.1 \mathrm{mM}$ EDTA, 0.1\% NP-40, 0.1\% glycerol, protease inhibitors cocktail) for $30 \mathrm{~min}$ on ice. The lysates were spun for $20 \mathrm{~min}$ at $14,000 \mathrm{rpm}$ to collect nuclear lysates. The following antibodies were used for western blot analysis: anti-Nanog (Cell Signaling Technology, 8822), anti-Oct4 (Cell Signaling Technology, 2840), anti-Klf4 (Cell Signaling Technology, 4038), anti-Pten (Cell Signaling Technology, 9188), anti- $\beta$-actin (Immunoway, YM3028), anti-p-T308-Akt (Cell Signaling Technology, 13038), anti-p-S473-Akt (Cell Signaling Technology, 4060), anti-Akt (Cell Signaling Technology, 4691), anti-pS9-Gsk3ß (Cell Signaling Technology, 5558), anti-Gsk3 (Cell Signaling Technology, 5676), anti-p-S380/T382/ T383-Pten (Cell Signaling Technology, 9554), anti$\beta$-catenin (Cell Signaling Technology, 8480), anti-Stat3 (Santa Cruz, sc483), anti-Rex1 antibody (Abcam, ab175429), anti-Gata4 antibody (Abcam, ab84593), anti-Mef2c antibody (Cell Signaling Technology, 5030), anti-p-Y705-Stat3 (Cell Signaling Technology, 9145), anti- $\beta$-Tubulin (Cell Signaling Technology, 2146), anti-pT389-p70 S6 Kinase (Cell Signaling Technology, 9234), anti-p70 S6 Kinase (Cell Signaling Technology, 9202), anti-p-S235/236-S6 Ribosomal protein (Cell Signaling Technology, 4858), anti-S6 Ribosomal protein (Cell Signaling Technology, 2217), anti-c-Myc (Cell Signaling Technology, 5605), anti-c-Jun (Cell Signaling Technology, 9165), anti-Sox2 (Cell Signaling Technology, 14962), antip-S250/251-Sox2 (Cell Signaling Technology, 92186), and anti-Lamin A/C (Cell Signaling Technology, 4777).

\section{Flow cytometry}

A total of $1 \times 10^{6}$ cells (WT ESCs, passage number 29) were required for optimal performance. The cells were 
fixed by adding $100 \mu \mathrm{l}$ of $4 \%$ PFA to each sample for $15 \mathrm{~min}$ and then permeabilized by adding $100 \mu \mathrm{l}$ of PBS and $0.3 \%$ Triton-X 100 . The cells were stained with primary and secondary antibodies. The primary antibody was anti-Rex1 antibody (Abcam, ab175429) and the secondary antibodies were donkey anti-mouse $\operatorname{IgG}(\mathrm{H}+\mathrm{L})$ Secondary Antibody (Life Technologies, A16018) and donkey anti-mouse IgG $\mathrm{H}+\mathrm{L}$ (TRITC) preadsorbed (Abcam, ab7058).

\section{Cell proliferation assay}

The number of viable cells was determined by a 3-(4,5dimethylthiazol-2-yl)-5-(3-carboxymethoxyphenyl)-2-(4sulfophenyl)-2H-tetrazolium (MTS) assay with a CellTiter 96 aqueous Cell Proliferation Assay kit (Promega).

\section{Cell apoptosis assay}

Cell apoptosis was detected by using a kit to the product manual (Invitrogen, V13241).

\section{Real-time PCR analysis}

Total RNA was isolated using Trizol (Invitrogen), and $500 \mathrm{ng}$ of RNA was reverse-transcribed using a HighCapacity cDNA Reverse Transcription Kit (Applied Biosystems). mRNA expression was measured by SYBR Green quantitative PCR using the ${ }^{\Delta \Delta}$ Ct method. Gapdh was used for normalization. Technical replicates were carried out for all quantitative PCR reactions, and the primer sequences are listed in Table S1.

\section{RNA-seq}

RNA-seq was performed for WT, Pten ${ }^{-/}$, and Pten-3A mutant ESCs and for WT and Pten $^{-1-}$ EBs. Total RNA was isolated from mouse ESCs and EBs according to the manufacturer's instructions using TRIzol reagent. The RNA was converted into a template molecule library for sequencing on the BGISEQ-500 (BGI, ShenZhen, China). Kyoto encyclopedia of genes and genomes pathway enrichment analysis was performed by the DAVID Functional Annotation Clustering Tool (DAVID 6.8, https://david.ncifcrf.gov).

\section{Statistics}

All statistical analyses were conducted using GraphPad Prism (version 6). Statistical significance was calculated by Student's $t$-test between the indicated groups. Significance was defined by $* p<0.05 ; * p<0.01$; and ${ }^{* * *} p<0.001$. All experiments were performed independently at least three times.

\section{Acknowledgements}

This work was supported by two Innovation and Technology Commission of Hong Kong government grants (PiH/078/18 and ITS/053/17FX) to CUHK; a Croucher Foundation CAS Joint Laboratory Scheme grant (CUHK-GIBH Joint Lab) (6904333); a National Natural Science Foundation of China (NSFC)/
Research Grants Council (RGC) Joint Research Scheme for 2016/17 (Ref No: N_CUHK415/16) and a general fund from CUHK-SDU Joint Laboratory on Reproductive Genetics, CUHK.

\section{Author details}

${ }^{1}$ CUHK-SDU Joint Laboratory on Reproductive Genetics, School of Biomedical Sciences, The Chinese University of Hong Kong, Hong Kong, China. ${ }^{2}$ National Research Center for Assisted Reproductive Technology and Reproductive Genetics, Jinan 250001, China. ${ }^{3}$ SDIVF R\&D Centre, Hong Kong Science Park, Shatin, Hong Kong, China. ${ }^{4}$ Center for Reproductive Medicine, Renji Hospital, School of Medicine, Shanghai Jiao Tong University, Shanghai 200135, China

\section{Data availability}

The RNA-seq raw data and normalized mapped reads are available from the Gene Expression Omnibus (GEO) at accession number: GSE117280. All other data supporting the findings of this study are available from the corresponding author on reasonable request.

\section{Conflict of interest}

The authors declare that they have no conflict of interest.

\section{Publisher's note}

Springer Nature remains neutral with regard to jurisdictional claims in published maps and institutional affiliations.

Supplementary Information accompanies this paper at (https://doi.org/ 10.1038/s41419-020-2271-0).

Received: 11 June 2019 Revised: 9 January 2020 Accepted: 15 January 2020 Published online: 07 February 2020

\section{References}

1. Thomson, J. A. et al. Embryonic stem cell lines derived from human blastocysts. Science 282, 1145-1147 (1998).

2. Martin, G. R. Isolation of a pluripotent cell line from early mouse embryos cultured in medium conditioned by teratocarcinoma stem cells. Proc. Natl Acad. Sci. USA 78, 7634-7638 (1981).

3. Reubinoff, B. E., Pera, M. F., Fong, C. Y., Trounson, A. \& Bongso, A. Embryonic stem cell lines from human blastocysts: somatic differentiation in vitro. Nat. Biotechnol. 18, 399-404 (2000).

4. Bradley, A., Evans, M., Kaufman, M. H. \& Robertson, E. Formation of germ-line chimaeras from embryo-derived teratocarcinoma cell lines. Nature 309, 255-256 (1984).

5. Hackett, J. A. \& Surani, M. A. Regulatory principles of pluripotency: from the ground state up. Cell Stem Cell 15, 416-430 (2014).

6. Hough, S. R. et al. Single-cell gene expression profiles define self-renewing, pluripotent, and lineage primed states of human pluripotent stem cells. Stem Cell Rep. 2, 881-895 (2014).

7. Sato, N., Meijer, L., Skaltsounis, L., Greengard, P. \& Brivanlou, A. H. Maintenance of pluripotency in human and mouse embryonic stem cells through activation of Wnt signaling by a pharmacological GSK-3-specific inhibitor. Nat. Med. 10, 55-63 (2004).

8. Tsutsui, H. et al. An optimized small molecule inhibitor cocktail supports longterm maintenance of human embryonic stem cells. Nat. Commun. 2, 167 (2011).

9. Ying, Q. L. et al. The ground state of embryonic stem cell self-renewal. Nature 453, 519-523 (2008).

10. Ying, Q. L., Nichols, J., Chambers, I. \& Smith, A. BMP induction of Id proteins suppresses differentiation and sustains embryonic stem cell self-renewal in collaboration with STAT3. Cell 115, 281-292 (2003).

11. Matsuda, T. et al. STAT3 activation is sufficient to maintain an undifferentiated state of mouse embryonic stem cells. EMBO J. 18, 4261-4269 (1999).

12. Di Cristofano, A., Pesce, B., Cordon-Cardo, C. \& Pandolfi, P. P. Pten is essential for embryonic development and tumour suppression. Nat. Genet. 19, 348-355 (1998).

13. Zheng, $\mathrm{H}$. et al. p53 and Pten control neural and glioma stem/progenitor cell renewal and differentiation. Nature 455, 1129-1133 (2008). 
14. Li, L., Liu, F. \& Ross, A. H. PTEN regulation of neural development and CNS stem cells. J. Cell. Biochem. 88, 24-28 (2003).

15. Alva, J. A., Lee, G. E., Escobar, E. E. \& Pyle, A. D. Phosphatase and tensin homolog regulates the pluripotent state and lineage fate choice in human embryonic stem cells. Stem Cells 29, 1952-1962 (2011).

16. Sun, $H$. et al. PTEN modulates cell cycle progression and cell survival by regulating phosphatidylinositol 3,4,5,-trisphosphate and Akt/protein kinase B signaling pathway. Proc. Natl Acad. Sci. USA 96, 6199-6204 (1999).

17. Zheng, W. J. \& Liu, K. The emerging role of maternal phosphatidylinositol 3 kinase (PI3K) signaling in manipulating mammalian preimplantation embryogenesis. Cell Cycle 10, 178-179 (2011).

18. Paling, N. R., Wheadon, H., Bone, H. K. \& Welham, M. J. Regulation of embryonic stem cell self-renewal by phosphoinositide 3-kinase-dependent signaling. J. Biol. Chem. 279, 48063-48070 (2004).

19. Armstrong, L. et al. The role of PI3K/AKT, MAPK/ERK and NFkappabeta signalling in the maintenance of human embryonic stem cell pluripotency and viability highlighted by transcriptional profiling and functional analysis. Hum. Mol. Genet. 15, 1894-1913 (2006).

20. Groszer, M. et al. PTEN negatively regulates neural stem cell self-renewal by modulating G0-G1 cell cycle entry. Proc. Natl Acad. Sci. USA 103, 111-116 (2006).

21. Dhara, S. K. \& Stice, S. L. Neural differentiation of human embryonic stem cells. J. Cell. Biochem. 105, 633-640 (2008).

22. Lu, Y. et al. The PTEN/MMAC1/TEP tumor suppressor gene decreases cell growth and induces apoptosis and anoikis in breast cancer cells. Oncogene 18, 7034-7045 (1999).

23. Groszer, M. et al. Negative regulation of neural stem/progenitor cell proliferation by the Pten tumor suppressor gene in vivo. Science 294, 2186-2189 (2001).

24. Nichols, J. \& Smith, A. Naive and primed pluripotent states. Cell Stem Cell 4, 487-492 (2009)

25. Cross, D. A., Alessi, D. R., Cohen, P., Andjelkovich, M. \& Hemmings, B. A. Inhibition of glycogen synthase kinase-3 by insulin mediated by protein kinase B. Nature 378, 785-789 (1995).

26. Cohen, P. \& Frame, S. The renaissance of GSK3. Nat. Rev. Mol. Cell Biol. 2 769-776 (2001).

27. Yilmaz, O. H. et al. Pten dependence distinguishes haematopoietic stem cells from leukaemia-initiating cells. Nature 441, 475-482 (2006).

28. Yokogami, K., Wakisaka, S., Avruch, J. \& Reeves, S. A. Serine phosphorylation and maximal activation of STAT3 during CNTF signaling is mediated by the rapamycin target mTOR. Curr. Biol. 10, 47-50 (2000).
29. Ling, L. S., Voskas, D. \& Woodgett, J. R. Activation of PDK-1 maintains mouse embryonic stem cell self-renewal in a PKB-dependent manner. Oncogene 32, 5397-5408 (2013).

30. Hay, N. The Akt-mTOR tango and its relevance to cancer. Cancer Cell $\mathbf{8}$, 179-183 (2005)

31. Inoki, K., Li, Y., Zhu, T. Q., Wu, J. \& Guan, K. L. TSC2 is phosphorylated and inhibited by Akt and suppresses mTOR signalling. Nat. Cell Biol. 4, 648-657 (2002).

32. Doble, B. W., Patel, S., Wood, G. A., Kockeritz, L. K. \& Woodgett, J. R. Functional redundancy of GSK-3 alpha and GSK-3 beta in Wnt/beta-catenin signaling shown by using an allelic series of embryonic stem cell lines. Dev. Cell 12, 957-971 (2007).

33. Dravid, G. et al. Defining the role of Wnt/beta-catenin signaling in the survival, proliferation, and self-renewal of human embryonic stem cells. Stem Cells $\mathbf{2 3}$, 1489-1501 (2005).

34. Ten Berge, D. et al. Embryonic stem cells require Wnt proteins to prevent differentiation to epiblast stem cells. Nat. Cell Biol. 13, 1070-U1088 (2011).

35. Ying, Q. L. et al. The ground state of embryonic stem cell self-renewal. Nature 453, 519-U515 (2008)

36. Lee, J. et al. Akt mediates self-renewal division of mouse spermatogonial stem cells. Development 134, 1853-1859 (2007).

37. Watanabe, S. et al. Activation of Akt signaling is sufficient to maintain pluripotency in mouse and primate embryonic stem cells. Oncogene $\mathbf{2 5}$ 2697-2707 (2006).

38. Li, Y. et al. Pretreatment with phosphatase and tensin homolog deleted on chromosome 10 (PTEN) inhibitor SF1670 augments the efficacy of granulocyte transfusion in a clinically relevant mouse model. Blood 117, 6702-6713 (2011).

39. Vazquez, F. et al. Phosphorylation of the PTEN tail acts as an inhibitory switch by preventing its recruitment into a protein complex. J. Biol. Chem. 276 48627-48630 (2001)

40. Torres, J. \& Pulido, R. The tumor suppressor PTEN is phosphorylated by the protein kinase CK2 at its C terminus. Implications for PTEN stability to proteasome-mediated degradation. J. Biol. Chem. 276, 993-998 (2001).

41. Vazquez, F., Ramaswamy, S., Nakamura, N. \& Sellers, W. R. Phosphorylation of the PTEN tail regulates protein stability and function. Mol. Cell. Biol. 20, 5010-5018 (2000)

42. Lee, J. H. et al. Lineage-specific differentiation is influenced by state of human pluripotency. Cell Rep. 19, 20-35 (2017).

43. Liao, J. et al. Inhibition of PTEN tumor suppressor promotes the generation of induced pluripotent stem cells. Mol. Ther. 21, 1242-1250 (2013). 